\title{
WELL-POSEDNESS FOR NON-ISOTROPIC DEGENERATE PARABOLIC-HYPERBOLIC EQUATIONS
}

\author{
Gui-Qiang CHEN ${ }^{\mathrm{a}}$, Benoît PERTHAME ${ }^{\mathrm{b}, *}$ \\ ${ }^{a}$ Department of Mathematics, Northwestern University, Evanston, IL 60208-2730, USA \\ ${ }^{\mathrm{b}}$ Département de mathématiques et applications, UMR 8553, École normale supérieure, 45, rue d'Ulm, \\ 75230 Paris cedex 05, France
}

Received 6 December 2001

In memory of Ph. Bénilan

ABSTRACT. - We develop a well-posedness theory for solutions in $L^{1}$ to the Cauchy problem of general degenerate parabolic-hyperbolic equations with non-isotropic nonlinearity. A new notion of entropy and kinetic solutions and a corresponding kinetic formulation are developed which extends the hyperbolic case. The notion of kinetic solutions applies to more general situations than that of entropy solutions; and its advantage is that the kinetic equations in the kinetic formulation are well defined even when the macroscopic fluxes are not locally integrable, so that $L^{1}$ is a natural space on which the kinetic solutions are posed. Based on this notion, we develop a new, simpler, more effective approach to prove the contraction property of kinetic solutions in $L^{1}$, especially including entropy solutions. It includes a new ingredient, a chain rule type condition, which makes it different from the isotropic case.

(C) 2003 L'Association Publications de l'Institut Henri Poincaré. Published by Elsevier B.V. All rights reserved

MSC: 35K65; 35K10; 35B30; 35D05

Keywords: Kinetic solutions; Entropy solutions; Kinetic formulation; Degenerate parabolic equations; Convection-diffusion; Non-isotropic diffusion; Stability; Existence; Well-posedness

RÉSUMÉ. - Nous développons une théorie d'existence et unicité pour les solutions $L^{1}$ seulement du problème de Cauchy pour un problème de Cauchy hyperbolique-parabolique avec diffusion non-isotrope générale. Des notions de formulations entropique et cinétique sont introduites qui incorporent un nouvel ingrédient, une condition de type dérivation composée, qui montre la différence fondamentale avec le cas d'une diffusion isotrope. L'avantage de la notion de solution cinétique est de travailler directement dans l'espace naturel $L^{1}$.

(C) 2003 L'Association Publications de l'Institut Henri Poincaré. Published by Elsevier B.V. All rights reserved

* Corresponding author.

E-mail addresses: gqchen@math.northwestern.edu (G.-Q.Chen), benoit.perthame@ens.fr

(B. Perthame). 


\section{Introduction and main theorems}

Consider the Cauchy problem of a general nonlinear degenerate parabolic-hyperbolic equation of second-order:

$$
\begin{gathered}
\partial_{t} u+\nabla \cdot f(u)=\nabla \cdot(A(u) \nabla u), \quad x \in \mathbb{R}^{d}, t \geqslant 0, \\
\left.u\right|_{t=0}=u_{0} \in L^{1}\left(\mathbb{R}^{d}\right),
\end{gathered}
$$

where $f: \mathbb{R} \rightarrow \mathbb{R}^{d}$ satisfies

$$
a(\cdot):=f^{\prime}(\cdot) \in L_{\mathrm{loc}}^{\infty}\left(\mathbb{R} ; \mathbb{R}^{d}\right),
$$

and the $d \times d$ matrix $A(u)=\left(a_{i j}(u)\right)$ is symmetric, nonnegative, and locally bounded so that we can always write

$$
a_{i j}(u)=\sum_{k=1}^{K} \sigma_{i k}(u) \sigma_{j k}(u), \quad \sigma_{i k} \in L_{\mathrm{loc}}^{\infty}(\mathbb{R}),
$$

and $\left(\sigma_{i k}(u)\right)$ is its square root matrix, in which the structure appears more naturally with the additional index $K$ that can be thought to be the maximal rank of the matrix. Equation (1.1) and its variants model degenerate diffusion-convection motions of ideal fluids and arise in a wide variety of important applications, including two phase flows in porous media (cf. [7] and the references cited therein) and sedimentation-consolidation processes (cf. [5] and the references cited therein). Since its importance in applications, there is a large literature for the design and analysis of various numerical methods to calculate solutions of $(1.1)$ and its variants; see $[7,12,11,9,17]$ and the references cited therein, for which a well-posedness theory for (1.1) is in great demand. We are concerned with the well-posedness, especially uniqueness and stability, for solutions of the Cauchy problem (1.1) and (1.2). The well-posedness issue is relatively well understood if one removes the diffusion term $\nabla \cdot(A(u) \nabla u)$, thereby obtaining a scalar hyperbolic conservation law; see Kruzhkov [18], Lions, Perthame and Tadmor [19,20], and Perthame [21,22]. It is equally well understood if one removes the convection term $\nabla \cdot f(u)$; see $[4,16]$ and the references cited therein. For the isotropic diffusion, $a_{i j}(u)=0, i \neq j$, some stability results for entropy solutions have been obtained for $B V$ solutions by Volpert and Hudjaev [24] in 1969. Only in 1999, Carrillo [6] could extend this result to $L^{\infty}$ solutions (also see Eymard et al. [14], Karlsen and Risebro [17] for further extensions), and Chen and DiBenedetto [8] handled the case of unbounded entropy solutions which may grow when $|x|$ is large. Also see Gilding [16] for a theory for isotropic degenerate parabolic equations with isolated degenerate points.

In this paper, we establish a well-posedness theory for $L^{1}$ solutions of the Cauchy problem (1.1) and (1.2) for general degenerate parabolic-hyperbolic equations of secondorder, especially including the non-isotropic diffusion case. This relies on two new ingredients. Firstly, the extension from the isotropic to the non-isotropic is not a purely technical issue and we introduce a fundamental and natural chain-rule type property, which does not appear in the isotropic case and which turns out to be the cornerstone for the uniqueness in the non-isotropic case. Secondly, we extend a notion of 
kinetic solutions, a new concept in this context, and a corresponding kinetic formulation. The notion of kinetic solutions applies to more general situations than that of entropy solutions as considered in [6,17] and [8]. The advantage of the new notion is that the kinetic equation in the kinetic formulation is well defined even when the macroscopic fluxes are not locally integrable so that $L^{1}$ is a natural space on which the kinetic solutions are posed. Based on this notion, the corresponding kinetic formulation and the uniqueness proof in the purely hyperbolic case introduced in [21], we develop a new, simpler, more effective approach, in comparison with the previous proofs in $[6,17]$ and [8], to prove the contraction property of kinetic solutions in $L^{1}$, especially including entropy solutions. This leads to a well-posedness theory for kinetic solutions in $L^{1}$ of the Cauchy problem of (1.1) and (1.2).

The main theorems of this paper are the following.

THEOREM 1.1. - Assume that (1.3) and (1.4) hold. Then

(i) For any kinetic solution $u \in L^{\infty}\left([0, \infty) ; L^{1}\left(\mathbb{R}^{d}\right)\right)$ with initial data $u_{0}(x)$, we have

$$
\left\|u(t)-u_{0}\right\|_{L^{1}\left(\mathbb{R}^{d}\right)} \rightarrow 0, \quad t \rightarrow 0 .
$$

(ii) If $u, v \in L^{\infty}\left([0, \infty) ; L^{1}\left(\mathbb{R}^{d}\right)\right)$ are kinetic solutions to (1.1) and (1.2) with initial data $u_{0}(x)$ and $v_{0}(x)$, respectively, then

$$
\|u(t)-v(t)\|_{L^{1}\left(\mathbb{R}^{d}\right)} \leqslant\left\|u_{0}-v_{0}\right\|_{L^{1}\left(\mathbb{R}^{d}\right)} .
$$

(iii) Furthermore, if $u \in L^{\infty}\left([0, \infty) \times \mathbb{R}^{d}\right)$, this kinetic solution is an entropy solution.

Theorem 1.2. - Assume that (1.3) and (1.4) hold. For $u_{0} \in L^{1}\left(\mathbb{R}^{d}\right)$, there exists a unique kinetic solution $u \in C\left([0, \infty) ; L^{1}\left(\mathbb{R}^{d}\right)\right)$ for the Cauchy problem (1.1) and (1.2). If $u_{0} \in L^{\infty} \cap L^{1}\left(\mathbb{R}^{d}\right)$, then the kinetic solution is the unique entropy solution and $|u(t, x)| \leqslant\left\|u_{0}\right\|_{L^{\infty}\left(\mathbb{R}^{d}\right)}$.

In Section 2, we derive a kinetic formulation in a precise manner and describe the notions of entropy solutions and kinetic solutions of the Cauchy problem (1.1) and (1.2). The new ingredient of this formulation is the precise identification of the kinetic defect measure and the degenerate parabolic defect measure, even in the region where $u(t, x)$ is discontinuous and is only in $L^{1}$. To make the points more clearly, in Section 3, we present our new approach by a formal proof to show the contraction property of kinetic solutions. Then Sections 4-6 are devoted to the rigorous proof of the stability of kinetic solutions. In Section 7, we prove the existence of kinetic solutions and entropy solutions of the Cauchy problem (1.1) and (1.2).

In this paper we focus on the prototypical case (1.1). The results and techniques straightforward extends to more general degenerate parabolic-hyperbolic equations of second order, by combining with the Gronwall inequality, such as

$$
\partial_{t} u+\nabla \cdot f(u, t, x)-\nabla \cdot(A(u, t, x) \nabla u)=c(u, t, x), \quad x \in \mathbb{R}^{d}, t \geqslant 0,
$$


where $A(u, t, x)=\left(a_{i j}(u, t, x)\right)$ with $a_{i j}(u, t, x)=a_{j i}(u, t, x), f(u, t, x)$, and $c(u, t, x)$ are sufficiently smooth functions, and

$$
\sum_{i, j} a_{i j}(u, t, x) \xi_{i} \xi_{j} \geqslant 0
$$

for $(t, x) \in \mathbb{R}_{+}^{d+1}$ and $u \in \mathbb{R}$.

\section{Entropy solutions, kinetic solutions, and kinetic formulation}

Eq. (1.1) satisfies a so-called entropy property. To motivate it, we consider a nondegenerate parabolic equation (1.1) in which the matrix $A(u)=\left(a_{i j}(u)\right)$ is replaced by $A(u)+\varepsilon I$, and we denote $u^{\varepsilon}(t, x)$ its $C^{2}$ solution. Then, for any function $S(\cdot) \in C^{2}(\mathbb{R})$, multiplying Eq. (1.1) by $S^{\prime}\left(u^{\varepsilon}\right)$ yields

$$
\begin{aligned}
& \partial_{t} S\left(u^{\varepsilon}\right)+\sum_{i=1}^{d} \partial_{x_{i}} \eta_{i}^{S}\left(u^{\varepsilon}\right)-\sum_{i, j=1}^{d} \partial_{x_{i}}\left(S^{\prime}\left(u^{\varepsilon}\right) a_{i j}\left(u^{\varepsilon}\right) \partial_{x_{j}} u^{\varepsilon}\right)-\varepsilon \Delta S\left(u^{\varepsilon}\right) \\
& =-m_{\varepsilon}^{S^{\prime \prime}}(t, x)-n_{\varepsilon}^{S^{\prime \prime}}(t, x),
\end{aligned}
$$

where the entropy flux $\eta_{i}^{S}(u)$ is defined (up to an additive constant) by

$$
\left(\eta_{i}^{S}\right)^{\prime}(u)=a_{i}(u) S^{\prime}(u),
$$

the entropy dissipation measure $m_{\varepsilon}^{S^{\prime \prime}}(t, x)$ is defined by

$$
m_{\varepsilon}^{S^{\prime \prime}}(t, x):=\varepsilon S^{\prime \prime}\left(u^{\varepsilon}\right)\left|\nabla u^{\varepsilon}\right|^{2} \geqslant 0
$$

and the parabolic dissipation measure $n_{\varepsilon}^{S^{\prime \prime}}(t, x)$ is given by

$$
n_{\varepsilon}^{S^{\prime \prime}}(t, x):=S^{\prime \prime}\left(u^{\varepsilon}\right) \sum_{i, j=1}^{d} a_{i j}\left(u^{\varepsilon}\right) \partial_{x_{i}} u^{\varepsilon} \partial_{x_{j}} u^{\varepsilon}=S^{\prime \prime}\left(u^{\varepsilon}\right) \sum_{k=1}^{K}\left(\sum_{i=1}^{d} \sigma_{i k}\left(u^{\varepsilon}\right) \partial_{x_{i}} u^{\varepsilon}\right)^{2} \geqslant 0 .
$$

In order to understand more about the dissipation measures, we introduce the notations $\beta_{i k}(u)$ and $\beta_{i k}^{\psi}(u)$ for $\psi \in C_{0}(\mathbb{R})$ with $\psi \geqslant 0$ :

$$
\beta_{i k}^{\prime}(u)=\sigma_{i k}(u), \quad\left(\beta_{i k}^{\psi}\right)^{\prime}(u)=\sqrt{\psi(u)} \sigma_{i k}(u) .
$$

Then we end up with the two equivalent definitions:

$$
n_{\varepsilon}^{\psi}(t, x):=\sum_{k=1}^{K}\left(\sum_{i=1}^{d} \partial_{x_{i}} \beta_{i k}^{\psi}\left(u^{\varepsilon}\right)\right)^{2}=\sum_{k=1}^{K} \psi\left(u^{\varepsilon}\right)\left(\sum_{i=1}^{d} \partial_{x_{i}} \beta_{i k}\left(u^{\varepsilon}\right)\right)^{2} .
$$

The heart of our investigations is to notice that this equality still holds in the limit $\varepsilon \rightarrow 0$.

It is useful at this stage to derive a priori bounds from the above calculations. After the space-time integration against $S^{\prime}$ with $S$ convex and $S^{\prime}(0)=S(0)=0$, we obtain 


$$
\begin{aligned}
& \int_{0}^{\infty} \int_{\mathbb{R}^{d}}\left(m_{\varepsilon}^{S^{\prime \prime}}(t, x)+n_{\varepsilon}^{S^{\prime \prime}}(t, x)\right) \mathrm{d} t \mathrm{~d} x \\
& \quad=\int_{0}^{\infty} \int_{\mathbb{R}^{d}} S^{\prime \prime}\left(u^{\varepsilon}\right)\left(\sum_{k=1}^{K}\left(\sum_{i=1}^{d} \partial_{x_{i}} \beta_{i k}\left(u^{\varepsilon}\right)\right)^{2}+\varepsilon\left|\nabla u^{\varepsilon}\right|^{2}\right) \mathrm{d} t \mathrm{~d} x \\
& \quad \leqslant\left\|S\left(u_{0}\right)\right\|_{L^{1}\left(\mathbb{R}^{d}\right)} \leqslant\left\|S^{\prime}\right\|_{L^{\infty}(\mathbb{R})}\left\|u_{0}\right\|_{L^{1}\left(\mathbb{R}^{d}\right)} .
\end{aligned}
$$

The following convenient notations are deduced by the duality $\left(C_{0}(\mathbb{R}) ; \mathcal{M}^{1}(\mathbb{R})\right)$, which replace the exponent $S^{\prime \prime}$ or $\psi$. Namely,

$$
m_{\varepsilon}^{\psi}(t, x)=\int_{\mathbb{R}} \psi(\xi) m_{\varepsilon}(t, x, \xi) \mathrm{d} \xi, \quad n_{\varepsilon}^{\psi}(t, x)=\int_{\mathbb{R}} \psi(\xi) n_{\varepsilon}(t, x, \xi) \mathrm{d} \xi,
$$

with

$$
\begin{gathered}
m_{\varepsilon}(t, x, \xi)=\delta\left(\xi-u^{\varepsilon}\right) \varepsilon\left|\nabla u^{\varepsilon}\right|^{2} \\
n_{\varepsilon}(t, x, \xi)=\delta\left(\xi-u^{\varepsilon}\right) \sum_{k=1}^{K}\left(\sum_{i=1}^{d} \partial_{x_{i}} \beta_{i k}\left(u^{\varepsilon}\right)\right)^{2},
\end{gathered}
$$

where $\delta(\xi)$ is the Dirac mass concentrated at $\xi=0$.

Then we can choose, as a limiting case for smoothness of the entropy $S(u)$, the function $S(u)=(u-\xi)_{+}$for the parameter $\xi \geqslant 0$, or $S(u)=(u-\xi)_{-}$for $\xi \leqslant 0$, in (2.5), and we end up with $\int_{0}^{\infty} \int_{\mathbb{R}^{d}}\left(m_{\varepsilon}+n_{\varepsilon}\right)(t, x, \xi) \mathrm{d} t \mathrm{~d} x \leqslant \mu(\xi) \in L_{0}^{\infty}(\mathbb{R})$ (bounded functions that vanish at infinity)

$$
\mu(\xi):=\mathbb{1}_{\{\xi>0\}}\left\|\left(u_{0}-\xi\right)_{+}\right\|_{L^{1}\left(\mathbb{R}^{d}\right)}+\mathbb{1}_{\{\xi<0\}}\left\|\left(u_{0}-\xi\right)_{-}\right\|_{L^{1}\left(\mathbb{R}^{d}\right)} .
$$

Choosing $S(u)=u^{2} / 2$, we also deduce from (2.5)

$$
\begin{aligned}
& \int_{0}^{\infty} \int_{\mathbb{R}^{d}}\left(m_{\varepsilon}+n_{\varepsilon}\right)(t, x, \xi) \mathrm{d} t \mathrm{~d} x \mathrm{~d} \xi \\
& \quad=\int_{0}^{\infty} \int_{\mathbb{R}^{d}}\left(\sum_{k=1}^{K}\left(\sum_{i=1}^{d} \partial_{x_{i}} \beta_{i k}\left(u^{\varepsilon}\right)\right)^{2}+\varepsilon\left|\nabla u^{\varepsilon}\right|^{2}\right) \mathrm{d} t \mathrm{~d} x \leqslant \frac{1}{2}\left\|u_{0}\right\|_{L^{2}\left(\mathbb{R}^{d}\right)} .
\end{aligned}
$$

As $\varepsilon \rightarrow 0$, passing to the limit with the above bounds and under the property that $u^{\varepsilon}(t, x)$ converges strongly (see Section 7 below), we end up with the definition of entropy solutions.

DEFINITION 2.1. - An entropy solution is a function $u(t, x) \in L^{\infty}\left([0, \infty) \times \mathbb{R}^{d}\right)$ such that

(i) $\sum_{i=1}^{d} \partial_{x_{i}} \beta_{i k}(u) \in L^{2}\left([0, \infty) \times \mathbb{R}^{d}\right)$, for any $k \in\{1, \ldots, K\}$;

(ii) for any function $\psi \in C_{0}(\mathbb{R})$ with $\psi(u) \geqslant 0$ and any $k \in\{1, \ldots, K\}$,

$$
\sum_{i=1}^{d} \partial_{x_{i}} \beta_{i k}^{\psi}(u)=\sqrt{\psi(u)} \sum_{i=1}^{d} \partial_{x_{i}} \beta_{i k}(u) \in L^{2}\left([0, \infty) \times \mathbb{R}^{d}\right),
$$


and

$$
\begin{aligned}
n^{\psi}(t, x) & :=\psi(u(t, x)) \sum_{k=1}^{K}\left(\sum_{i=1}^{d} \partial_{x_{i}} \beta_{i k}(u(t, x))\right)^{2} \\
& =\sum_{k=1}^{K}\left(\sum_{i=1}^{d} \partial_{x_{i}} \beta_{i k}^{\psi}(u(t, x))\right)^{2} \text { a.e.; }
\end{aligned}
$$

(iii) for any smooth function $S(u)$, there exists an entropy dissipation measure $m^{S^{\prime \prime}}(t, x)$ satisfying that

$$
m^{S^{\prime \prime}}(t, x)=\int_{\mathbb{R}} S^{\prime \prime}(\xi) m(t, x, \xi) \mathrm{d} \xi, \quad \text { with } m(t, x, \xi) \text { a nonnegative measure, }
$$

such that

$$
\partial_{t} S(u)+\sum_{i=1}^{d} \partial_{x_{i}} \eta_{i}^{S}(u)-\sum_{i, j=1}^{d} \partial_{x_{i}}\left(a_{i j}(u) \partial_{x_{j}} S(u)\right)=-\left(m^{S^{\prime \prime}}+n^{S^{\prime \prime}}\right),
$$

in $\mathcal{D}^{\prime}\left(\mathbb{R}^{+} \times \mathbb{R}^{d}\right)$ with initial data $S(u(t=0))=S\left(u_{0}\right)$.

Remark 2.1. - Arguing as in (2.7), an entropy solution satisfies

$$
\int_{0}^{\infty} \int_{\mathbb{R}^{d}}(m+n)(t, x, \xi) \mathrm{d} t \mathrm{~d} x \leqslant \mu(\xi) \in L_{0}^{\infty}(\mathbb{R}) .
$$

Remark 2.2. - The nonnegative parabolic defect measure $n(t, x, \xi)$ for an entropy solution $u(t, x)$ in Definition 2.1 is very simple and given by the following formula:

$$
n(t, x, \xi)=\delta(\xi-u(t, x)) \sum_{k=1}^{K}\left(\sum_{i=1}^{d} \partial_{x_{i}} \beta_{i k}(u(t, x))\right)^{2},
$$

in the usual sense. Also, the choice $S(u)=u^{2} / 2$ gives the $L^{2}$-integrability of $\sum_{i=1}^{d} \partial_{x_{i}} \beta_{i k}(u), 1 \leqslant k \leqslant K$, and yields another useful estimate, as in (2.8):

$$
\int_{0}^{\infty} \int_{\mathbb{R}^{d+1}}(m+n)(t, x, \xi) \mathrm{d} t \mathrm{~d} x \mathrm{~d} \xi \leqslant \frac{1}{2}\left\|u_{0}\right\|_{L^{2}\left(\mathbb{R}^{d}\right)}^{2},
$$

provided $u_{0} \in L^{2}\left(\mathbb{R}^{d}\right)$.

Remark 2.3. - When we refer to distributional solutions here, we always mean that the initial data are included in the definition of solutions in the sense of distributions, when a test function does not vanish at $t=0$. That is, a distributional solution $u(t, x)$ satisfying (2.12) means that, for any test function $\varphi \in \mathcal{D}\left([0, \infty) \times \mathbb{R}^{d}\right)$, 


$$
\begin{gathered}
\int_{0}^{\infty} \int_{\mathbb{R}^{d}}\left(S(u) \partial_{t} \varphi+\eta^{S}(u) \cdot \nabla_{x} \varphi-\sum_{i, j=1}^{d} \alpha_{i j}^{S^{\prime}}(u) \partial_{x_{i} x_{j}}^{2} \varphi\right) \mathrm{d} t \mathrm{~d} x \\
=\int_{0}^{\infty} \int_{\mathbb{R}^{d}}\left(m^{S^{\prime \prime}}+n^{S^{\prime \prime}}\right) \varphi \mathrm{d} t \mathrm{~d} x-\int_{\mathbb{R}^{d}} S\left(u_{0}(x)\right) \varphi(0, x) \mathrm{d} x,
\end{gathered}
$$

with $\left(\alpha_{i j}^{S^{\prime}}\right)^{\prime}(u)=S^{\prime}(u) a_{i j}(u)$. However, thanks to the chain rule which is postulated in the definition of entropy solutions, several possible variants for the second-order term are equivalent.

Remark 2.4. - The main ingredient in Definition 2.1 is the equality in (2.9), which is not always true for a function $u(t, x)$. Indeed, if $\beta_{i k}(u)$ is discontinuous, this chain rule does not make sense even for any single term in the sums of (2.9). It is natural to assume the equality here because it keeps true in the limiting process $u^{\varepsilon}(t, x) \rightarrow u(t, x)$ strongly (see Section 7). In the case of a diagonal matrix $a_{i j}=0$ for $i \neq j$, this equality in (2.9) is always true and needs not be included in Definition 2.1. We refer to the appendix for a proof. Therefore, our theory also recovers the results of Carrillo [6] (and the extensions of $[17,14])$ and Chen and DiBenedetto [8] when the initial data are in $L^{1} \cap L^{\infty}$.

On the other hand, we may factor out an $S^{\prime}(u)$ in the equation (2.12) and obtain a more precise kinetic formulation of nonlinear degenerate parabolic-hyperbolic equations of second-order with form (1.1). The new ingredient of this formulation is the identification of the kinetic defect measure $m(t, x, \xi)$ and the degenerate parabolic defect measure $n(t, x, \xi)$ in a precise manner, even in the region where $u(t, x)$ is discontinuous and only in $L^{1}$. Compare with the classical kinetic formulation for multidimensional hyperbolic conservation laws by Lions, Perthame and Tadmor in $[19,20]$.

We introduce the kinetic function $\chi$ on $\mathbb{R}^{2}$ :

$$
\chi(\xi ; u)= \begin{cases}+1 & \text { for } 0<\xi<u \\ -1 & \text { for } u<\xi<0 \\ 0 & \text { otherwise }\end{cases}
$$

We notice that, if $u \in L^{\infty}\left([0, \infty) ; L^{1}\left(\mathbb{R}^{d}\right)\right)$, then $\chi(\xi ; u) \in L^{\infty}\left([0, \infty) ; L^{1}\left(\mathbb{R}^{d+1}\right)\right)$.

The simple representation

$$
S(u)=\int_{\mathbb{R}} S^{\prime}(\xi) \chi(\xi ; u) \mathrm{d} \xi
$$

leads to the following kinetic equation, which is equivalent to (2.12):

$$
\partial_{t} \chi(\xi ; u)+a(\xi) \cdot \nabla_{x} \chi(\xi ; u)-\sum_{i, j=1}^{d} a_{i j}(\xi) \partial_{x_{i} x_{j}}^{2} \chi(\xi ; u)=\partial_{\xi}(m+n)(t, x, \xi)
$$

in $\mathcal{D}^{\prime}\left(\mathbb{R}^{+} \times \mathbb{R}^{d+1}\right)$ with initial data

$$
\left.\chi(\xi ; u)\right|_{t=0}=\chi\left(\xi ; u_{0}\right)
$$


We are now ready to define the kinetic solutions.

Definition 2.2. - A kinetic solution is a function $u(t, x) \in L^{\infty}\left([0, \infty) ; L^{1}\left(\mathbb{R}^{d}\right)\right)$ such that

(i) for any nonnegative $\psi \in \mathcal{D}(\mathbb{R})$ and $k \in\{1, \ldots, K\}$,

$$
\sum_{i=1}^{d} \partial_{x_{i}} \beta_{i k}^{\psi}(u) \in L^{2}\left([0, \infty) \times \mathbb{R}^{d}\right)
$$

(ii) for any two nonnegative functions $\psi_{1}, \psi_{2} \in \mathcal{D}(\mathbb{R})$,

$$
\sqrt{\psi_{1}(u(t, x))} \sum_{i=1}^{d} \partial_{x_{i}} \beta_{i k}^{\psi_{2}}(u(t, x))=\sum_{i=1}^{d} \partial_{x_{i}} \beta_{i k}^{\psi_{1} \psi_{2}}(u(t, x)) \text { a.e.; }
$$

(iii) Eq. (2.16) holds in $\mathcal{D}^{\prime}$, for some nonnegative measures $m(t, x, \xi)$ and $n(t, x, \xi)$, where $n(t, x, \xi)$ is defined by

$$
\int_{\mathbb{R}} \psi(\xi) n(t, x, \xi) \mathrm{d} \xi=\sum_{k=1}^{K}\left(\sum_{i=1}^{d} \partial_{x_{i}} \beta_{i k}^{\psi}(u(t, x))\right)^{2}, \quad \text { for any } \psi \in \mathcal{D}(\mathbb{R}) \text { with } \psi \geqslant 0
$$

(iv) the following inequality is satisfied:

$$
\int_{0}^{\infty} \int_{\mathbb{R}^{d}}(m+n)(t, x, \xi) \mathrm{d} t \mathrm{~d} x \leqslant \mu(\xi) \in L_{0}^{\infty}(\mathbb{R}) .
$$

This notion of kinetic solutions applies to more general situations than that of entropy solutions. The advantage is that the kinetic equation is well defined even though the macroscopic fluxes $\eta^{S}(u)$ are not locally integrable so that $L^{1}$ is a natural space on which kinetic solutions are posed. In the purely hyperbolic case, a full $L^{1}$-theory has been developed in Perthame [22]. This approach also covers the so-called renormalized solutions used in the context of hyperbolic scalar conservation laws by Bénilan, Carrillo, and Wittbold [1].

Remark 2.5. - Any entropy solution is a kinetic solution. Our uniqueness result implies that any kinetic solution in $L^{\infty}$ must be an entropy solution. Therefore, the two notions are equivalent for solutions in $L^{\infty}$, although the notion of kinetic solutions is more general.

Remark 2.6. - The degenerate parabolic defect measure $n(t, x, \xi)$ is no longer defined by the simple formula in Remark 2.2 since $\sum_{i=1}^{d} \partial_{x_{i}} \beta_{i k}(u)$ does not belong to $L^{2}\left([0, \infty) \times \mathbb{R}^{d}\right)$ in general because (2.14) does not apply. In fact, the only a priori bound used here is that of (2.13) which is also expressed in Remark 2.1 as a corollary of Definition 2.1. The explicit expression in terms of $u_{0}(x)$ for $\mu(\xi)$ in (2.7) is not fundamental, and the useful information is that $\mu(\xi)$ is bounded and vanishes at infinity. 


\section{Contraction proof: formal}

In this section, we give a formal proof for the contraction property of kinetic solutions, i.e., part (ii) of Theorem 1.1, which takes the advantage of the precise kinetic formulation (2.15)-(2.20). We will make the proof rigorous in Sections 4-6.

Consider two solutions $u(t, x)$ and $v(t, x)$. Denote by $p(t, x, \xi)$ the kinetic defect measure and by

$$
q(t, x, \xi):=\delta(\xi-v(t, x)) \sum_{k=1}^{K}\left(\sum_{i=1}^{d} \partial_{x_{i}} \beta_{i k}(v(t, x))\right)^{2},
$$

the parabolic defect measure, which are associated with $v(t, x)$. Then our proof consists in using the following microscopic contraction functional introduced in [21,22]:

$$
Q(t, x, \xi)=|\chi(\xi ; u(t, x))|+|\chi(\xi ; v(t, x))|-2 \chi(\xi ; u(t, x)) \chi(\xi ; v(t, x)) \geqslant 0
$$

It is useful for deriving a contraction principle since

$$
\int_{\mathbb{R}^{d}} Q(t, x, \xi) \mathrm{d} \xi=|u(t, x)-v(t, x)| .
$$

The point is to justify the following identities. Firstly,

$$
\begin{aligned}
& \partial_{t}|\chi(\xi ; u(t, x))|+a(\xi) \cdot \nabla_{x}|\chi(\xi ; u(t, x))|-\sum_{i, j=1}^{d} \partial_{x_{i} x_{j}}^{2}\left(a_{i j}(\xi)|\chi(\xi ; u(t, x))|\right) \\
& \quad=\operatorname{sgn}(\xi) \partial_{\xi}(m+n)(t, x, \xi)
\end{aligned}
$$

which yields

$$
\frac{\mathrm{d}}{\mathrm{d} t} \int_{\mathbb{R}^{d+1}}|\chi(\xi ; u(t, x))| \mathrm{d} x \mathrm{~d} \xi=-2 \int_{\mathbb{R}^{d}}(m+n)(t, x, 0) \mathrm{d} x .
$$

A similar identity holds for $v(t, x)$.

Secondly, we compute

$$
\begin{aligned}
& \frac{\mathrm{d}}{\mathrm{d} t} \int_{\mathbb{R}^{d+1}} \chi(\xi ; u(t, x)) \chi(\xi ; v(t, x)) \mathrm{d} x \mathrm{~d} \xi \\
& \quad+2 \int_{\mathbb{R}^{d+1}} \sum_{i, j=1}^{d} a_{i j}(\xi) \partial_{x_{i}} \chi(\xi ; u(t, x)) \partial_{x_{j}} \chi(\xi ; v(t, x)) \mathrm{d} x \mathrm{~d} \xi \\
& \quad=\int_{\mathbb{R}^{d+1}}((m+n)(t, x, \xi)(\delta(\xi-v(t, x))-\delta(\xi)) \\
& \quad+(p+q)(t, x, \xi)(\delta(\xi-u(t, x))-\delta(\xi))) \mathrm{d} x \mathrm{~d} \xi .
\end{aligned}
$$

Then, we have 


$$
\begin{aligned}
& \frac{\mathrm{d}}{\mathrm{d} t} \int_{\mathbb{R}^{d+1}} Q(t, x, \xi) \mathrm{d} x \mathrm{~d} \xi \\
& \quad=4 \int_{\mathbb{R}^{d+1}} \sum_{i, j=1}^{d} a_{i j}(\xi) \partial_{x_{i}} \chi(\xi ; u(t, x)) \partial_{x_{j}} \chi(\xi ; v(t, x)) \mathrm{d} x \mathrm{~d} \xi \\
& \quad-2 \int_{\mathbb{R}^{d+1}}((m+n)(t, x, \xi) \delta(\xi-v(t, x))+(p+q)(t, x, \xi) \delta(\xi-u(t, x))) \mathrm{d} x \mathrm{~d} \xi \\
& \quad 4 \int_{\mathbb{R}^{d+1}} \sum_{i, j=1}^{d} a_{i j}(\xi) \partial_{x_{i}} u(t, x) \partial_{x_{j}} v(t, x) \delta(\xi-u(t, x)) \delta(\xi-v(t, x)) \mathrm{d} x \mathrm{~d} \xi \\
& \quad-2 \int_{\mathbb{R}^{d+1}}(n(t, x, \xi) \delta(\xi-v(t, x))+q(t, x, \xi) \delta(\xi-u(t, x))) \mathrm{d} x \mathrm{~d} \xi,
\end{aligned}
$$

since $m(t, x, \xi)$ and $p(t, x, \xi)$ are nonnegative.

It remains to notice that, using Remark 2.2 and (3.1), we still have, very formally,

$$
\begin{aligned}
\int_{\mathbb{R}^{d+1}}( & n(t, x, \xi) \delta(\xi-v(t, x))+q(t, x, \xi) \delta(\xi-u(t, x))) \mathrm{d} x \mathrm{~d} \xi \\
= & \sum_{k=1}^{K} \int_{\mathbb{R}^{d+1}} \delta(\xi-u(t, x)) \delta(\xi-v(t, x)) \\
& \left.\times\left(\sum_{i=1}^{d} \partial_{x_{i}} \beta_{i k}(u(t, x))\right)^{2}+\left(\sum_{i=1}^{d} \partial_{x_{i}} \beta_{i k}(v(t, x))\right)^{2}\right) \mathrm{d} x \mathrm{~d} \xi \\
\geqslant & 2 \sum_{k=1}^{K} \int_{\mathbb{R}^{d+1}} \delta(\xi-u(t, x)) \delta(\xi-v(t, x)) \\
& \times\left(\sum_{i=1}^{d} \partial_{x_{i}} \beta_{i k}(u(t, x))\right)\left(\sum_{j=1}^{d} \partial_{x_{j}} \beta_{j k}(v(t, x))\right) \mathrm{d} x \mathrm{~d} \xi \\
= & 2 \sum_{k=1}^{K} \sum_{i, j=1}^{d} \int_{\mathbb{R}^{d+1}} \delta(\xi-u(t, x)) \delta(\xi-v(t, x)) \sigma_{i k}(u(t, x)) \\
& \times \sigma_{j k}(v(t, x)) \partial_{x_{i}} u(t, x) \partial_{x_{j}} v(t, x) \mathrm{d} x \mathrm{~d} \xi \\
= & 2 \sum_{i, j=1}^{d} \int_{\mathbb{R}^{d+1}} a_{i j}(\xi) \partial_{x_{i}} u(t, x) \partial_{x_{j}} u(t, x) \delta(\xi-u(t, x)) \delta(\xi-v(t, x)) \mathrm{d} x \mathrm{~d} \xi
\end{aligned}
$$

Therefore, we end up with

$$
\frac{\mathrm{d}}{\mathrm{d} t} \int_{\mathbb{R}^{d+1}} Q(t, x, \xi) \mathrm{d} x \mathrm{~d} \xi \leqslant 0
$$


which implies that $\|u(t)-v(t)\|_{L^{1}\left(\mathbb{R}^{d}\right)}$ is non-increasing. This concludes the contraction property (1.5).

\section{Contraction proof: rigorous}

To make the proof rigorous, the above argument requires to regularize the linear kinetic equation (2.16) by convolution; This is the first step, in which all notations are also introduced. Then we analyze separately the different terms in the microscopic contraction functional, which requires several steps.

Step 1. Regularization. We set $\varepsilon=\left(\varepsilon_{1}, \varepsilon_{2}\right), \varepsilon_{1}$ for the forward time regularization and $\varepsilon_{2}$ for the space regularization, and we define

$$
\varphi_{\varepsilon}(t, x)=\frac{1}{\varepsilon_{1}} \varphi_{1}\left(\frac{t}{\varepsilon_{1}}\right) \frac{1}{\varepsilon_{2}^{d}} \varphi_{2}\left(\frac{x}{\varepsilon_{2}}\right),
$$

where $\varphi_{j} \geqslant 0, j=1,2$, denote the normalized regularizing kernels with $\int \varphi_{j}=1$, $\operatorname{supp}\left(\varphi_{1}\right) \subset(-1,0)$ in order to allow the time regularization.

Next, we use the notations

$$
\begin{aligned}
\chi:=\chi(t, x, \xi)=\chi(\xi ; u(t, x)), & \tilde{\chi}:=\tilde{\chi}(\xi ; t, x)=\chi(\xi ; v(t, x)), \\
\chi_{\varepsilon}:=\chi_{\varepsilon}(t, x, \xi)=\left(\chi *_{(t, x)} \varphi_{\varepsilon}\right)(t, x, \xi), & \tilde{\chi}_{\varepsilon}:=\tilde{\chi}_{\varepsilon}(t, x, \xi)=\left(\tilde{\chi} *_{(t, x)} \varphi_{\varepsilon}\right)(t, x, \xi),
\end{aligned}
$$

and, similarly,

$$
m_{\varepsilon}:=m *_{(t, x)} \varphi_{\varepsilon}, \quad p_{\varepsilon}:=p *_{(t, x)} \varphi_{\varepsilon}, \quad n_{\varepsilon}:=n *_{(t, x)} \varphi_{\varepsilon}, \quad q_{\varepsilon}:=q *_{(t, x)} \varphi_{\varepsilon},
$$

where $*_{(t, x)}$ denotes the convolution in time and space. We also need a further regularization in $\xi$ with smoothing kernel $\psi_{\delta}(\xi)=\frac{1}{\delta} \psi\left(\frac{\xi}{\delta}\right)$ and use the notation

$$
\chi_{\varepsilon, \delta}:=\chi_{\varepsilon} * \psi_{\delta}
$$

Finally, we need a $\xi$-truncation $K_{R}(\xi)$, which is a smooth nonnegative function with bounded support. That is, $K_{R}(\xi)=K(\xi / R) \rightarrow 1$, as $R \rightarrow \infty$, with

$$
\begin{array}{ll}
0 \leqslant K(\xi) \leqslant 1 & \text { for } \xi \in(-\infty, \infty), \\
K(\xi)=1 & \text { for }|\xi| \leqslant 1 / 2, \\
K(\xi)=0 & \text { for }|\xi| \geqslant 1 .
\end{array}
$$

The destiny of these parameters is that $\delta \rightarrow 0$ first, $R \rightarrow \infty$ second, and $\varepsilon \rightarrow 0$ finally. The results we will show indicate that the contraction property holds even for any fixed parameter $\varepsilon$, which is an interesting phenomenon. Indeed, for any regularized microscopic contraction functional:

$$
Q_{\varepsilon}(t, x, \xi)=\left|\chi_{\varepsilon}\right|+\left|\tilde{\chi}_{\varepsilon}\right|-2 \chi_{\varepsilon} \tilde{\chi}_{\varepsilon} \geqslant 0,
$$

we have 
PROPOSITION 4.1. - Under the assumptions of Theorem 1.1,

$$
\frac{\mathrm{d}}{\mathrm{d} t} \int_{\mathbb{R}^{d+1}} Q_{\varepsilon}(t, x, \xi) \mathrm{d} x \mathrm{~d} \xi \leqslant 0 .
$$

Notice that, by convolution, we obtain

$$
\begin{aligned}
& \partial_{t} \chi_{\varepsilon}+a(\xi) \cdot \nabla_{x} \chi_{\varepsilon}-\sum_{i, j=1}^{d} \partial_{x_{i} x_{j}}^{2}\left(a_{i j}(\xi) \chi_{\varepsilon}\right)=\partial_{\xi}\left(m_{\varepsilon}+n_{\varepsilon}\right)(t, x, \xi), \\
& \partial_{t} \tilde{\chi}_{\varepsilon}+a(\xi) \cdot \nabla_{x} \tilde{\chi}_{\varepsilon}-\sum_{i, j=1}^{d} \partial_{x_{i} x_{j}}^{2}\left(a_{i j}(\xi) \tilde{\chi}_{\varepsilon}\right)=\partial_{\xi}\left(p_{\varepsilon}+q_{\varepsilon}\right)(t, x, \xi)
\end{aligned}
$$

in $\mathcal{D}^{\prime}\left((0, \infty) \times \mathbb{R}^{d+1}\right)$, where the initial data are inessential at this stage.

Step 2. First terms of the contraction functional. From the space and time regularity of $\chi_{\varepsilon}$, we deduce that $\left(m_{\varepsilon}+n_{\varepsilon}\right)(t, x, \xi)$ is locally Lipschitz continuous in $\xi$. Furthermore, multiplying (4.2) by $\operatorname{sgn}(\xi)$, we find

$$
\frac{\mathrm{d}}{\mathrm{d} t} \int_{\mathbb{R}^{d+1}}\left|\chi_{\varepsilon}(\xi ; t, x)\right| \mathrm{d} x \mathrm{~d} \xi=-2 \int_{\mathbb{R}^{d}}\left(m_{\varepsilon}+n_{\varepsilon}\right)(t, x, 0) \mathrm{d} x .
$$

Similarly,

$$
\frac{\mathrm{d}}{\mathrm{d} t} \int_{\mathbb{R}^{d+1}}\left|\tilde{\chi}_{\varepsilon}(\xi ; t, x)\right| \mathrm{d} x \mathrm{~d} \xi=-2 \int_{\mathbb{R}^{d}}\left(p_{\varepsilon}+q_{\varepsilon}\right)(t, x, 0) \mathrm{d} x .
$$

Step 3. Quadratic term of the contraction functional. Analyzing the quadratic term requires a further $\xi$-regularization. We write

$$
\partial_{t} \chi_{\varepsilon, \delta}+a(\xi) \cdot \nabla_{x} \chi_{\varepsilon, \delta}-\sum_{i, j=1}^{d} \partial_{x_{i} x_{j}}^{2}\left(\left(a_{i j}(\xi) \chi_{\varepsilon}\right) * \psi_{\delta}\right)=\partial_{\xi}\left(\left(m_{\varepsilon}+n_{\varepsilon}\right) * \psi_{\delta}\right)+R_{1}^{u},
$$

with

$$
R^{u}=R_{1}^{u}(t, x, \xi):=\operatorname{div}_{x}\left(a(\xi)\left(\chi_{\varepsilon} * \psi_{\delta}\right)-\left(a(\xi) \chi_{\varepsilon}\right) * \psi_{\delta}\right) .
$$

A similar equation holds for $\tilde{\chi}_{\varepsilon, \delta}$. Therefore, a direct combination gives

$$
\begin{aligned}
& \partial_{t}\left(\tilde{\chi}_{\varepsilon, \delta} \chi_{\varepsilon, \delta} K_{R}(\xi)\right)+K_{R}(\xi) a(\xi) \cdot \nabla_{x}\left(\tilde{\chi}_{\varepsilon, \delta} \chi_{\varepsilon, \delta}\right)-K_{R}(\xi) \tilde{\chi}_{\varepsilon, \delta} \sum_{i, j=1}^{d} \partial_{x_{i} x_{j}}^{2}\left(\left(a_{i j}(\xi) \chi_{\varepsilon}\right) * \psi_{\delta}\right) \\
& \quad-K_{R}(\xi) \chi_{\varepsilon, \delta} \sum_{i, j=1}^{d} \partial_{x_{i} x_{j}}^{2}\left(\left(a_{i j}(\xi) \tilde{\chi}_{\varepsilon}\right) * \psi_{\delta}\right) \\
& =\tilde{\chi}_{\varepsilon, \delta} K_{R}(\xi) \partial_{\xi}\left(\left(m_{\varepsilon}+n_{\varepsilon}\right) * \psi_{\delta}\right)+\tilde{\chi}_{\varepsilon, \delta} K_{R}(\xi) R_{1}^{u}+\chi_{\varepsilon, \delta} K_{R}(\xi) \partial_{\xi}\left(\left(p_{\varepsilon}+q_{\varepsilon}\right) * \psi_{\delta}\right) \\
& \quad+\chi_{\varepsilon, \delta} K_{R}(\xi) R_{1}^{v}
\end{aligned}
$$


After integration, we obtain

$$
\frac{\mathrm{d}}{\mathrm{d} t} \int_{\mathbb{R}^{d+1}} \tilde{\chi}_{\varepsilon, \delta} \chi_{\varepsilon, \delta} K_{R}(\xi) \mathrm{d} x \mathrm{~d} \xi=\sum_{l=1}^{3} \mathcal{R}_{l}(t)+\sum_{l=4}^{7}\left(\mathcal{R}_{l}(t)+\mathcal{R}_{l}^{\prime}(t)\right)
$$

where $R_{l}$ are defined as follows:

$$
\begin{gathered}
\mathcal{R}_{1}(t)=\int_{\mathbb{R}^{d+1}} K_{R}(\xi)\left(\tilde{\chi}_{\varepsilon, \delta} R_{1}^{u}+\chi_{\varepsilon, \delta} R_{1}^{v}\right) \mathrm{d} x \mathrm{~d} \xi \\
\mathcal{R}_{2}(t)=-2 \int_{\mathbb{R}^{d+1}} K_{R}(\xi) \sum_{k=1}^{K} \sum_{i, j=1}^{d} \partial_{x_{i}}\left(\left(\sigma_{i k} \chi_{\varepsilon}\right) * \psi_{\delta}\right) \partial_{x_{j}}\left(\left(\sigma_{j k} \tilde{\chi}_{\varepsilon}\right) * \psi_{\delta}\right) \mathrm{d} x \mathrm{~d} \xi \\
\mathcal{R}_{3}(t)=-\int_{\mathbb{R}^{d+1}} K_{R}(\xi) \psi_{\delta}(\xi)\left(\left(m_{\varepsilon}+n_{\varepsilon}\right) * \psi_{\delta}+\left(p_{\varepsilon}+q_{\varepsilon}\right) * \psi_{\delta}\right) \mathrm{d} x \mathrm{~d} \xi \\
\mathcal{R}_{4}(t)=\int_{\mathbb{R}^{d+1}} K_{R}(\xi) \sum_{i, j=1}^{d}\left(-\partial_{x_{i}} \tilde{\chi}_{\varepsilon, \delta} \partial_{x_{j}}\left(\left(a_{i j}(\xi) \chi_{\varepsilon}\right) * \psi_{\delta}\right)\right. \\
\left.+\partial_{x_{j}}\left(\sigma_{j k}(\xi) \chi_{\varepsilon, \delta} * \psi_{\delta}\right) \partial_{x_{i}}\left(\left(\sigma_{i k}(\xi) \tilde{\chi}_{\varepsilon}\right) * \psi_{\delta}\right)\right) \mathrm{d} x \mathrm{~d} \xi
\end{gathered}
$$

The term $\mathcal{R}_{5}(t)$ comes from integration by parts in $\xi$ and the following equality:

$$
\partial_{\xi} \chi_{\varepsilon, \delta}=\psi_{\delta}(\xi)-\delta(\xi-u) *_{(t, x, \xi)}\left(\varphi_{\varepsilon} \psi_{\delta}\right),
$$

that is, taking into account $\mathcal{R}_{3}(t)$,

$$
\mathcal{R}_{5}(t)=-\int_{\mathbb{R}^{d+1}} K_{R}^{\prime}(\xi) \tilde{\chi}_{\varepsilon, \delta}\left(\left(m_{\varepsilon}+n_{\varepsilon}\right) * \psi_{\delta}\right) \mathrm{d} x \mathrm{~d} \xi
$$

Also,

$$
\begin{aligned}
& \mathcal{R}_{6}(t)=\int_{\mathbb{R}^{d+1}} K_{R}(\xi)\left(\delta(\xi-v) *_{(t, x, \xi)}\left(\varphi_{\varepsilon} \psi_{\delta}\right)\right)\left(n_{\varepsilon} * \psi_{\delta}\right) \mathrm{d} x \mathrm{~d} \xi \\
& \mathcal{R}_{7}(t)=\int_{\mathbb{R}^{d+1}} K_{R}(\xi)\left(\delta(\xi-v) *_{(t, x, \xi)}\left(\varphi_{\varepsilon} \psi_{\delta}\right)\right)\left(m_{\varepsilon} * \psi_{\delta}\right) \mathrm{d} x \mathrm{~d} \xi .
\end{aligned}
$$

The terms $\mathcal{R}_{l}^{\prime}(t), 4 \leqslant l \leqslant 7$, denote the symmetric terms of $\mathcal{R}_{l}(t), 4 \leqslant l \leqslant 7$, respectively, where $u(t, x)$ is replaced by $v(t, x)$.

Step 4. Estimates of the error terms. Our goal is now to estimate these error terms in the following two lemmas.

LEMMA 4.1. - For any $1 \leqslant p<\infty$, when $\delta \rightarrow 0$,

$$
\mathcal{R}_{1}(t), \mathcal{R}_{4}(t), \mathcal{R}_{4}^{\prime}(t) \rightarrow 0, \quad L_{\mathrm{loc}}^{p}\left(\mathbb{R}_{+}\right)
$$


and

$$
\mathcal{R}_{3}(t) \rightarrow \int_{\mathbb{R}^{d}}\left(m_{\varepsilon}+n_{\varepsilon}\right)(t, x, 0) \mathrm{d} x+\int_{\mathbb{R}^{d}}\left(p_{\varepsilon}+q_{\varepsilon}\right)(t, x, 0) \mathrm{d} x, \quad L_{\mathrm{loc}}^{p}\left(\mathbb{R}_{+}\right) .
$$

In addition, when $\delta \rightarrow 0$ first and $R \rightarrow \infty$ second,

$$
\mathcal{R}_{5}(t), \mathcal{R}_{5}^{\prime}(t) \rightarrow 0, \quad \text { in } L_{\mathrm{loc}}^{p}\left(\mathbb{R}_{+}\right)
$$

Furthermore, for any $\varepsilon, \delta, R$,

$$
\mathcal{R}_{7}(t), \mathcal{R}_{7}^{\prime}(t) \geqslant 0, \quad \text { for any } t \in(0, \infty) .
$$

It remains to estimate the remaining terms which are more difficult to handle and contain the actual cancellation which motivates our kinetic formulation with the introduction of the parabolic defect measure $n(t, x, \xi)$.

LEMMA 4.2. - For any $\varepsilon, \delta, R$,

$$
\mathcal{R}_{2}(t)+\mathcal{R}_{6}(t)+\mathcal{R}_{6}^{\prime}(t) \geqslant 0, \quad \text { for any } t \in(0, \infty) .
$$

Lemma 4.1 is proved in Section 6.1. Lemma 4.2 is proved in Section 6.2 for entropy solutions (the easier case) and in Section 6.3 for kinetic solutions.

Step 5. Contraction property. With Lemmas 4.1 and 4.2, we can now conclude the contraction proof. As a consequence of the above lemmas, we can pass to the limit as $\delta \rightarrow 0$ first and $R \rightarrow \infty$ second in (4.4), (4.5), and (4.6). Adding (4.4), (4.5), and substracting twice (4.6) yields

$$
\frac{\mathrm{d}}{\mathrm{d} t} \int_{\mathbb{R}^{d+1}}\left(\left|\chi_{\varepsilon}\right|+\left|\tilde{\chi}_{\varepsilon}\right|-2 \tilde{\chi}_{\varepsilon} \chi_{\varepsilon}\right) \mathrm{d} x \mathrm{~d} \xi \leqslant 0
$$

This complete the proof of Proposition 4.1.

Since, when $\varepsilon \rightarrow 0$,

$$
\begin{aligned}
& \int_{\mathbb{R}^{d}}|u(t)-v(t)| \mathrm{d} x \\
& \quad=\int_{\mathbb{R}^{d+1}}(|\chi(\xi ; u(t, x))|+|\chi(\xi ; v(t, x))|-2 \chi(\xi ; u(t, x)) \chi(\xi ; v(t, x))) \mathrm{d} x \mathrm{~d} \xi,
\end{aligned}
$$

we conclude the contraction property, that is,

$$
\int_{\mathbb{R}^{d}}|u(t)-v(t)| \mathrm{d} x
$$

is non-increasing in $t>0$. The full result (ii) of Theorem 1.1 is therefore proved after using (i) which is proved below. 


\section{Continuity at $t=0$ and $L^{1}$-stability}

In order to obtain the $L^{1}$-stability (1.5), it remains to prove the initial time continuity statement (i) in Theorem 1.1. The proof, first due to [13] in the hyperbolic case, is based on the use of the initial data for all entropies in the weak form of Eq. (2.12). At the kinetic level, it amounts to say that $\chi(\xi ; u(t, x))$ achieves the initial data $\chi\left(\xi ; u_{0}\right)$ in the weak form of Eq. (2.16) (see Remark 2.3). We prove only for kinetic solutions which contains the case of entropy solutions and turns out to be rather simple.

Proposition 5.1.- Let $u_{0} \in L^{1}\left(\mathbb{R}^{d}\right)$ and $u(t, x)$ be a kinetic solution (see Definition 2.2). Then, when $t \rightarrow 0^{+}$,

$$
\int_{\mathbb{R}^{d}}\left|u(t, x)-u_{0}(x)\right| \mathrm{d} x \rightarrow 0,
$$

and

$$
\int_{0}^{t} \int_{\mathbb{R}^{d}} \int_{-L}^{L}(m+n)(s, x, \xi) \mathrm{d} s \mathrm{~d} x \mathrm{~d} \xi \rightarrow 0, \quad \text { for any } L>0 .
$$

Proof. - We follow the proof in [22] and thus we skip some technical details. Using the Dunford-Petti theorem, we first consider a nonnegative, strictly convex function $\Phi(u)$ with superlinear growth for large $|u|$ such that $\Phi(0)=0$ and $\int_{\mathbb{R}^{d}} \Phi\left(u_{0}(x)\right) \mathrm{d} x<+\infty$. Definition 2.2 for kinetic solutions yields the following identity:

$$
\int_{\mathbb{R}^{d+1}} \Phi(u(t, x)) \mathrm{d} x+\int_{0}^{t} \int_{\mathbb{R}^{d+1}} \Phi^{\prime \prime}(\xi)(m+n)(s, x, \xi) \mathrm{d} s \mathrm{~d} x \mathrm{~d} \xi=\int_{\mathbb{R}^{d}} \Phi\left(u_{0}(x)\right) \mathrm{d} x<\infty .
$$

This identity can be achieved by first choosing admissible test functions $\varphi_{n}(t, x) \Phi^{\prime}(\xi) \times$ $K(\xi / R)$ with $\varphi_{n}(t, x) \rightarrow \mathbb{1}_{\left\{0 \leqslant t \leqslant T, x \in \mathbb{R}^{d}\right\}}$, for any $T \in(0, \infty)$, and a smooth function $K(\xi)$ such that $K(\xi)=1$ for $|\xi|<1, K(\xi)=0$ for $|\xi| \geqslant 2$, and $\operatorname{sgn}(\xi) K(\xi) \leqslant 0$, and then taking $n \rightarrow \infty$ first and $R \rightarrow \infty$ second.

From (5.1) and the nonnegative sign of $m, n$, and $\Phi^{\prime \prime}$, we know that $u(t, x)$ is relatively weakly compact in $L_{\text {loc }}^{1}\left(\mathbb{R}^{d}\right)$. Hence we may extract subsequences such that $t_{n} \rightarrow 0$ and

$$
\begin{gathered}
\chi\left(\xi ; u\left(t_{n}, x\right)\right) \stackrel{*}{\rightarrow} \bar{\chi}(x, \xi) \in L^{1}\left(\mathbb{R}^{d+1}\right) \quad \text { in } L^{\infty}\left(\mathbb{R}^{d+1}\right), \\
u\left(t_{n}, x\right) \rightarrow \int_{\mathbb{R}} \bar{\chi}(x, \xi) \mathrm{d} \xi \quad \text { in } L_{\mathrm{loc}}^{1}\left(\mathbb{R}^{d}\right), \\
\int_{0}^{t_{n}}(m+n)(s, x, \xi) \mathrm{d} s \rightarrow \bar{m}(x, \xi) \quad \text { in } \mathcal{M}^{1}\left(\mathbb{R}^{d+1}\right),
\end{gathered}
$$

and

$$
\operatorname{sgn}(\xi) \bar{\chi}(x, \xi)=|\bar{\chi}(x, \xi)| \leqslant 1, \quad \bar{m}(x, \xi) \geqslant 0, \quad \int_{\mathbb{R}^{d}} \bar{m}(x, \xi) \mathrm{d} x \leqslant \mu(\xi) \in L_{0}^{\infty}(\mathbb{R}),
$$


where the half arrows " $\longrightarrow$ " and " $\stackrel{*}{\rightarrow}$ " denote the weak convergence and the weak-star convergence in the respective spaces, respectively, and $\mu(\xi)$ is the function defined in (2.13) and (2.20).

Secondly, using the definition of distributional solutions yields that, for any test function $\phi(x, \xi)$ in $\mathcal{D}$,

$$
\begin{aligned}
\int_{\mathbb{R}^{d+1}} \phi(x, \xi) \chi(\xi ; u(T, x)) \mathrm{d} x \mathrm{~d} \xi \\
\quad-\int_{0}^{T} \int_{\mathbb{R}^{d+1}}\left(a(\xi) \cdot \nabla_{x} \phi(x, \xi)-\sum_{i, j=1}^{d} a_{i j}(\xi) \partial_{x_{i} x_{j}} \phi(x, \xi)\right) \chi(\xi ; u(t, x)) \mathrm{d} t \mathrm{~d} x \mathrm{~d} \xi \\
=-\int_{0}^{T} \int_{\mathbb{R}^{d+1}} \phi_{\xi}(x, \xi)(m+n)(t, x, \xi) \mathrm{d} t \mathrm{~d} x \mathrm{~d} \xi+\int_{\mathbb{R}^{d+1}} \phi(x, \xi) \chi\left(\xi ; u_{0}(x)\right) \mathrm{d} x \mathrm{~d} \xi .
\end{aligned}
$$

Passing to the limit as $T=t_{n} \rightarrow 0$, we deduce that

$$
\begin{aligned}
\int_{\mathbb{R}^{d+1}} \phi(x, \xi) \bar{\chi}(x, \xi) \mathrm{d} x \mathrm{~d} \xi= & -\int_{\mathbb{R}^{d+1}} \phi_{\xi}(x, \xi) \bar{m}(x, \xi) \mathrm{d} x \mathrm{~d} \xi \\
& +\int_{\mathbb{R}^{d+1}} \phi(x, \xi) \chi\left(\xi ; u_{0}(x)\right) \mathrm{d} x \mathrm{~d} \xi,
\end{aligned}
$$

which implies that the identity holds in the sense of distributions:

$$
\partial_{\xi} \bar{m}(x, \xi)=\bar{\chi}(x, \xi)-\chi\left(\xi ; u_{0}(x)\right) .
$$

From this, we deduce that the measure $\bar{m}(x, \xi)$ is also a function and that, for almost every $x, \bar{m}(x, \cdot)$ is continuous and vanishes at infinity therefore $\int_{\mathbb{R}} \bar{\chi} \mathrm{d} \xi=u_{0}$. On the other hand, we know $\bar{m}(x, \xi) \geqslant 0$. Therefore, we deduce from Brenier's Lemma [3] that $\bar{m}(x, \xi)=0$ and $\bar{\chi}(x, \xi)=\chi\left(\xi ; u_{0}(x)\right)$. By the uniqueness of the limit, the whole family $\chi(\xi ; u(t, x))$ converges weak-star to $\chi\left(\xi ; u_{0}(x)\right)$ as $t \rightarrow 0$.

Therefore, $\chi(\xi ; u(t, x))$ has a trace $\chi(\cdot ; u(0+, \cdot))=\chi\left(\cdot ; u_{0}(\cdot)\right)$ on the set $t=0$, defined at least in the weak sense in $L^{1}\left(\mathbb{R}^{d+1}\right)$.

On the other hand, we also deduce that, for the locally strictly convex function $\Phi$ with superlinear growth at infinity in Step 1,

$$
\int_{\mathbb{R}^{d}} \Phi(u(t, x)) \mathrm{d} x \leqslant \int_{\mathbb{R}^{d}} \Phi\left(u_{0}(x)\right) \mathrm{d} x .
$$

The strict convexity of $\Phi$ implies that the trace of $u(t, x)$ on the set $t=0$ is in fact defined in the strong sense in $L^{1}$ as $t \rightarrow 0$ :

$$
u(t, x) \rightarrow u_{0}(x), \quad \text { in } L^{1}\left(\mathbb{R}^{d}\right),
$$


and, as a corollary,

$$
\int_{0}^{t} \int_{\mathbb{R}^{d+1}} \Phi^{\prime \prime}(\xi)(m+n)(s, x, \xi) \mathrm{d} s \mathrm{~d} x \mathrm{~d} \xi \rightarrow 0 .
$$

This completes the proof of Proposition 5.1.

\section{Proof of Lemmas 4.1 and 4.2}

In this section, we give the proof of Lemmas 4.1 and 4.2.

\subsection{Proof of Lemma 4.1}

Statement (4.16) is a simple consequence of the nonnegativity of the convolution and truncation kernels and the dissipation measures $m(t, x, \xi)$ and $p(t, x, \xi)$.

For the term $\mathcal{R}_{1}(t)$, it suffices to consider the representative term:

$$
\begin{aligned}
& \left|\int_{\mathbb{R}^{d+1}} K_{R}(\xi) \tilde{\chi}_{\varepsilon, \delta} \operatorname{div}_{x}\left(a(\xi) \chi_{\varepsilon} * \psi_{\delta}-\left(a(\xi) \chi_{\varepsilon}\right) * \psi_{\delta}\right) \mathrm{d} x \mathrm{~d} \xi\right| \\
& \quad=\left|\int_{\mathbb{R}^{d+1}} K_{R}(\xi) \tilde{\chi}_{\varepsilon, \delta}\left(a(\xi) \nabla_{x} \chi_{\varepsilon} * \psi_{\delta}-\left(a(\xi) \nabla_{x} \chi_{\varepsilon}\right) * \psi_{\delta}\right) \mathrm{d} x \mathrm{~d} \xi\right| \\
& \quad \leqslant \int_{\mathbb{R}^{d+2}} K_{R}(\xi)\left|\tilde{\chi}_{\varepsilon, \delta}(t, x, \xi)\right||a(\xi)-a(\xi-\eta)|\left|\nabla_{x} \chi_{\varepsilon}(t, x, \xi-\eta)\right| \psi_{\delta}(\eta) \mathrm{d} x \mathrm{~d} \xi \mathrm{d} \eta \\
& \quad \leqslant \frac{C\left(\varphi_{2}\right)}{\varepsilon_{2}} \int_{\mathbb{R}^{d+1}}\left|\tilde{\chi}_{\varepsilon, \delta}(t, x, \xi)\right|\left(\int_{\mathbb{R}} K_{R}(\xi)|a(\xi)-a(\xi-\eta)| \psi_{\delta}(\eta) \mathrm{d} \eta\right) \mathrm{d} x \mathrm{~d} \xi .
\end{aligned}
$$

Notice that the function of $\xi$ defined as

$$
\int_{\mathbb{R}} K_{R}(\xi)|a(\xi)-a(\xi-\eta)| \psi_{\delta}(\eta) \mathrm{d} \eta
$$

has a uniform bound $2\|a\|_{L^{\infty}(-R, R)}$ and tends to zero for a.e. $\xi$, when $\delta \rightarrow 0$. On the other hand, $\tilde{\chi}_{\varepsilon, \delta}(t, x, \xi)$ is compact in $L_{\text {loc }}^{p}\left((0, \infty) ; L^{1}\left(\mathbb{R}^{d} \times(-R, R)\right)\right)$. Therefore, by the Lebesgue Theorem, the expression (6.1) tends to zero in $L_{\mathrm{loc}}^{p}(0, \infty)$, for all $1 \leqslant p<\infty$. This proves the first statement of (4.13).

The fact that the term $\mathcal{R}_{4}$ tends to zero in $L_{\text {loc }}^{p}(0, \infty)$ can be obtained by following the same argument. Indeed, we have

$$
\begin{aligned}
\left|\mathcal{R}_{4}(t)\right| \leqslant & \int_{\mathbb{R}^{d+3}} K_{R}(\xi) \sum_{k=1}^{K} \sum_{i, j=1}^{d}\left|\partial_{x_{i}} \tilde{\chi}_{\varepsilon}(t, x, \xi-\eta)\right|\left|\partial_{x_{j}} \chi_{\varepsilon}\left(t, x, \xi-\eta^{\prime}\right)\right| \\
& \times\left|a_{i j}\left(\xi-\eta^{\prime}\right)-\sigma_{i k}(\xi-\eta) \sigma_{i k}\left(\xi-\eta^{\prime}\right)\right| \psi_{\delta}(\eta) \psi_{\delta}\left(\eta^{\prime}\right) \mathrm{d} x \mathrm{~d} \xi \mathrm{d} \eta \mathrm{d} \eta^{\prime}
\end{aligned}
$$




$$
\begin{aligned}
\leqslant & \frac{C\left(\varphi_{2}\right)}{\varepsilon_{2}} \int_{\mathbb{R}^{d+1}} \sum_{k=1}^{K} \sum_{i, j=1}^{d}\left|\partial_{x_{j}} \chi_{\varepsilon}\left(t, x, \xi-\eta^{\prime}\right)\right| \\
& \times\left(\int_{\mathbb{R}^{2}} K_{R}(\xi)\left|a_{i j}\left(\xi-\eta^{\prime}\right)-\sigma_{i k}(\xi-\eta) \sigma_{j k}\left(\xi-\eta^{\prime}\right)\right| \psi_{\delta}(\eta) \psi_{\delta}\left(\eta^{\prime}\right) \mathrm{d} \eta \mathrm{d} \eta^{\prime}\right) \mathrm{d} x \mathrm{~d} \xi,
\end{aligned}
$$

and we conclude as before.

The results (4.14) and (4.15) concerning $\mathcal{R}_{3}(t)$ and $\mathcal{R}_{5}(t)$ are much simpler because $\left(m_{\varepsilon}+n_{\varepsilon}\right)(t, x, \xi)$ and $\left(p_{\varepsilon}+q_{\varepsilon}\right)(t, x, \xi)$ are continuous in $\xi$, vanish at infinity, and their total masses are dominated by $\mu(\xi)$, because of (2.13). Therefore, we omit the proof.

\subsection{Proof of Lemma 4.2: entropy solutions}

In the case of entropy solutions, the definition of the measure $n(t, x, \xi)$ allows to write directly the expression $\mathcal{R}_{6}(t)+\mathcal{R}_{6}^{\prime}(t)$ with explicit convolution terms. This yields

$$
\begin{aligned}
\mathcal{R}_{6}+ & \mathcal{R}_{6}^{\prime} \\
= & \sum_{k=1}^{K} \int K_{R}(\xi) \varphi_{\varepsilon}(t-s, x-y) \varphi_{\varepsilon}\left(t-s^{\prime}, x-y^{\prime}\right) \psi_{\delta}(\xi-u(s, y)) \psi_{\delta}\left(\xi-v\left(s^{\prime}, y^{\prime}\right)\right) \\
& \times\left(\left|\sum_{i=1}^{d} \partial_{y_{i}} \beta_{i k}(u(s, y))\right|^{2}+\left|\sum_{j=1}^{d} \partial_{y_{j}} \beta_{j k}\left(v\left(s^{\prime}, y^{\prime}\right)\right)\right|^{2}\right) \mathrm{d} s \mathrm{~d} s^{\prime} \mathrm{d} x \mathrm{~d} y \mathrm{~d} y^{\prime} \mathrm{d} \xi \\
\geqslant & 2 \sum_{k=1}^{K} \int K_{R}(\xi) \varphi_{\varepsilon}(t-s, x-y) \varphi_{\varepsilon}\left(t-s^{\prime}, x-y^{\prime}\right) \psi_{\delta}(\xi-u(s, y)) \psi_{\delta}\left(\xi-v\left(s^{\prime}, y^{\prime}\right)\right) \\
& \times \sum_{i=1}^{d} \partial_{y_{i}} \beta_{i k}(u(s, y)) \sum_{j=1}^{d} \partial_{y_{j}} \beta_{j k}\left(v\left(s^{\prime}, y^{\prime}\right)\right) \mathrm{d} s \mathrm{~d} s^{\prime} \mathrm{d} x \mathrm{~d} y \mathrm{~d} y^{\prime} \mathrm{d} \xi .
\end{aligned}
$$

On the other hand, we have

$$
\begin{aligned}
\mathcal{R}_{2}(t)= & -2 \sum_{k=1}^{K} \sum_{i, j=1}^{d} \int K_{R}(\xi) \partial_{x_{i}} \varphi_{\varepsilon}(t-s, x-y) \partial_{x_{j}} \varphi_{\varepsilon}\left(t-s^{\prime}, x-y^{\prime}\right) \psi_{\delta}(\xi-\eta) \\
& \times \psi_{\delta}\left(\xi-\eta^{\prime}\right) \sigma_{i k}(\eta) \chi(\eta ; u(s, y)) \\
& \times \sigma_{j k}\left(\eta^{\prime}\right) \chi\left(\eta^{\prime} ; v\left(s^{\prime}, y^{\prime}\right)\right) \mathrm{d} s \mathrm{~d} s^{\prime} \mathrm{d} x \mathrm{~d} y \mathrm{~d} y^{\prime} \mathrm{d} \xi \mathrm{d} \eta \mathrm{d} \eta^{\prime}
\end{aligned}
$$

Consider for instance the following term from $\mathcal{R}_{2}(t)$ :

$$
\begin{aligned}
& \sum_{i=1}^{d} \int \partial_{x_{i}} \varphi_{\varepsilon}(t-s, x-y) \psi_{\delta}(\xi-\eta) \sigma_{i k}(\eta) \chi(\eta ; u(s, y)) \mathrm{d} \eta \mathrm{d} y \\
& \quad=\sum_{i=1}^{d} \int \partial_{x_{i}} \varphi_{\varepsilon}(t-s, x-y) \beta_{i k}^{\psi_{\delta}(\xi-\cdot)^{2}}(u(s, y)) \mathrm{d} y \\
& \quad=\sum_{i=1}^{d} \int \varphi_{\varepsilon}(t-s, x-y) \partial_{y_{i}} \beta_{i k}^{\psi_{\delta}(\xi-\cdot)^{2}}(u(s, y)) \mathrm{d} y,
\end{aligned}
$$


where the first equality follows from the definition in (2.3). This term is also equal to the following term from $\mathcal{R}_{6}(t)+\mathcal{R}_{6}^{\prime}(t)$ :

$$
\int \varphi_{\varepsilon}(t-s, x-y) \psi_{\delta}(\xi-u(s, y)) \sum_{i=1}^{d} \partial_{y_{i}} \beta_{i k}(u(s, y)) \mathrm{d} y,
$$

as a consequence of the identity between $L^{2}$ functions:

$$
\sum_{i=1}^{d} \partial_{y_{i}} \beta_{i k}^{\psi_{\delta}(\xi-)^{2}}(u(s, y))=\psi_{\delta}(\xi-u(s, y)) \sum_{i=1}^{d} \partial_{x_{i}} \beta_{i k}(u(s, y)),
$$

which is exactly statement (ii) in Definition 2.1 of entropy solutions.

Hence, Lemma 4.2 is proved for entropy solutions.

\subsection{Proof of Lemma 4.2: kinetic solutions}

When treating the kinetic solutions, we write again the expression $\mathcal{R}_{6}(t)+\mathcal{R}_{6}^{\prime}(t)$, but we only have at hand a weaker form than that in Section 6.2. That is,

$$
\begin{aligned}
\mathcal{R}_{6}(t)+\mathcal{R}_{6}^{\prime}(t)= & \sum_{k=1}^{K} \int K_{R}(\xi) \varphi_{\varepsilon}(t-s, x-y) \varphi_{\varepsilon}\left(t-s^{\prime}, x-y^{\prime}\right) \\
& \times\left(\psi_{\delta}\left(\xi-v\left(s^{\prime}, y^{\prime}\right)\right)\left|\sum_{i=1}^{d} \partial_{y_{i}} \beta_{i k}^{\psi_{\delta}(\xi-\cdot)}(u(s, y))\right|^{2}\right. \\
& \left.+\psi_{\delta}(\xi-u(s, y))\left|\sum_{j=1}^{d} \partial_{y_{j}} \beta_{j k}^{\psi_{\delta}(\xi-\cdot)}\left(v\left(s^{\prime}, y^{\prime}\right)\right)\right|^{2}\right) \mathrm{d} s \mathrm{~d} s^{\prime} \mathrm{d} x \mathrm{~d} y \mathrm{~d} y^{\prime} \mathrm{d} \xi \\
\geqslant & 2 \sum_{k=1}^{K} \int K_{R}(\xi) \varphi_{\varepsilon}(t-s, x-y) \varphi_{\varepsilon}\left(t-s^{\prime}, x-y^{\prime}\right) \\
& \times \sqrt{\psi_{\delta}(\xi-u(s, y))} \sqrt{\psi_{\delta}\left(\xi-v\left(s^{\prime}, y^{\prime}\right)\right)} \\
& \times \sum_{i=1}^{d} \partial_{y_{i}} \beta_{i k}^{\psi_{\delta}(\xi-\cdot)}(u(s, y)) \sum_{j=1}^{d} \partial_{y_{j}} \beta_{j k}^{\psi_{\delta}(\xi-\cdot)}\left(v\left(s^{\prime}, y^{\prime}\right)\right) \mathrm{d} s \mathrm{~d} s^{\prime} \mathrm{d} x \mathrm{~d} y \mathrm{~d} y^{\prime} \mathrm{d} \xi \\
= & 2 \sum_{k=1}^{K} \sum_{i, j=1}^{d} \int K_{R}(\xi) \varphi_{\varepsilon}(t-s, x-y) \varphi_{\varepsilon}\left(t-s^{\prime}, x-y^{\prime}\right) \\
& \times \sqrt{\psi_{\delta}(\xi-u(s, y))} \sqrt{\psi_{\delta}\left(\xi-v\left(s^{\prime}, y^{\prime}\right)\right)} \\
& \times \partial_{x_{i}} \beta_{i k}^{\psi_{\delta}(\xi-\cdot)}(u(s, y)) \partial_{x_{j}} \beta_{j k}^{\psi_{\delta}(\xi-\cdot)}\left(v\left(s^{\prime}, y^{\prime}\right)\right) \mathrm{d} s \mathrm{~d} s^{\prime} \mathrm{d} x \mathrm{~d} y \mathrm{~d} y^{\prime} \mathrm{d} \xi .
\end{aligned}
$$

On the other hand, the expression (6.2) for $\mathcal{R}_{2}(t)$ still holds. Therefore, we conclude the proof of Lemma 4.2 if we can justify, for instance,

$$
\int \partial_{x_{i}} \varphi_{\varepsilon}(t-s, x-y) \psi_{\delta}(\eta-u(s, y)) \sigma_{i k}(\xi-\eta) \chi(\xi-\eta ; u(s, y)) \mathrm{d} y \mathrm{~d} \eta
$$




$$
=\int \varphi_{\varepsilon}(t-s, x-y) \sqrt{\psi_{\delta}(\xi-u(s, y))} \partial_{x_{i}} \beta_{i k}^{\psi_{\delta}(\xi-\cdot)}(u(s, y)) \mathrm{d} y .
$$

This is exactly assumptions (2.17) and (2.18) in Definition 2.2. This concludes the proof of Lemma 4.2 for kinetic solutions.

\section{Existence of kinetic solutions}

We now prove Theorem 1.2 for the existence of kinetic solutions, especially entropy solutions, of the Cauchy problem (1.1) and (1.2).

We divide the proof into four steps.

Step 1 . We first consider the case $u_{0} \in W^{2,1} \cap H^{1} \cap L^{\infty}\left(\mathbb{R}^{d}\right)$ and we prove the existence of an entropy solution. To do so, we set

$$
\left(a_{i j}^{\varepsilon}(u)\right):=\left(a_{i j}(u)\right)+\varepsilon I .
$$

Then, Volpert-Hudjaev's theorem [24] implies that, for each $\varepsilon>0$, there is a smooth solution $u^{\varepsilon}(t, x)$ such that, for $t>0$, we have

$$
\begin{gathered}
\left\|u^{\varepsilon}(t, \cdot)\right\|_{L^{1}\left(\mathbb{R}^{d}\right)} \leqslant\left\|u_{0}\right\|_{L^{1}\left(\mathbb{R}^{d}\right)}, \quad\left\|u^{\varepsilon}(t, \cdot)\right\|_{L^{\infty}\left(\mathbb{R}^{d}\right)} \leqslant\left\|u_{0}\right\|_{L^{\infty}\left(\mathbb{R}^{d}\right)}, \\
\left\|\nabla_{x} u^{\varepsilon}(t, \cdot)\right\|_{L^{1}\left(\mathbb{R}^{d}\right)} \leqslant\left\|u_{0}\right\|_{T V\left(\mathbb{R}^{d}\right)}, \\
\left\|\partial_{t} u^{\varepsilon}(t, \cdot)\right\|_{L^{1}\left(\mathbb{R}^{d}\right)} \leqslant\left\|\partial_{t} u_{0}\right\|_{L^{1}\left(\mathbb{R}^{d}\right)}=\left\|\nabla \cdot f\left(u_{0}\right)-\nabla \cdot\left(A^{\varepsilon}\left(u_{0}\right) \nabla u_{0}\right)\right\|_{L^{1}\left(\mathbb{R}^{d}\right)},
\end{gathered}
$$

and inequalities (2.5) and (2.8) hold.

Then, for any $\Psi^{\prime \prime}=\psi \in \mathcal{D}_{+}, \psi^{\prime}(0)=\psi(0)=0$,

$$
\begin{aligned}
& \sum_{k=1}^{K} \int_{0}^{\infty} \int_{\mathbb{R}^{d}} \psi\left(u^{\varepsilon}\right)\left(\sum_{i=1}^{d} \partial_{x_{i}} \beta_{i k}\left(u^{\varepsilon}\right)\right)^{2} \mathrm{~d} x \mathrm{~d} t \\
& \quad=\sum_{i, j} \int_{0}^{\infty} \int_{\mathbb{R}^{d}} \psi\left(u^{\varepsilon}\right) a_{i j}^{\varepsilon}\left(u^{\varepsilon}\right) \partial_{x_{i}} u^{\varepsilon} \partial_{x_{j}} u^{\varepsilon} \mathrm{d} x \mathrm{~d} t \leqslant \int_{\mathbb{R}^{d}} \Psi\left(u_{0}^{\varepsilon}\right) \mathrm{d} x \leqslant C<\infty .
\end{aligned}
$$

By Kolmogorov's compactness theorem and after a standard control of decay at infinity, there is a subsequence (still denoted) $u^{\varepsilon}(t, x)$ such that

$$
u^{\varepsilon} \rightarrow u \quad \text { in } C_{\mathrm{loc}}\left([0, \infty) ; L^{1}\left(\mathbb{R}^{d}\right)\right)
$$

and thanks to (2.8),

$$
\sum_{i=1}^{d} \partial_{x_{i}} \beta_{i k}\left(u^{\varepsilon}\right) \rightarrow \sum_{i=1}^{d} \partial_{x_{i}} \beta_{i k}(u) \quad \text { in } L^{2}\left([0,+\infty) \times \mathbb{R}^{d}\right) .
$$

In particular, from this, we obtain property (i) of Definition 2.1

$$
\sum_{i=1}^{d} \partial_{x_{i}} \beta_{i k}(u) \in L^{2}\left([0,+\infty) \times \mathbb{R}^{d}\right)
$$


and,

$$
\sum_{k=1}^{K} \int_{0}^{\infty} \int_{\mathbb{R}^{d}}\left(\sum_{i}^{d} \partial_{x_{i}} \beta_{i k}(u)\right)^{2} \mathrm{~d} x \mathrm{~d} t \leqslant \frac{1}{2}\left\|u_{0}\right\|_{L^{2}\left(\mathbb{R}^{d}\right)}^{2} .
$$

Furthermore, for any nonnegative $\psi \in \mathcal{D}$ and $k \in\{1, \ldots, K\}$, we have

$$
\sum_{i=1}^{d} \partial_{x_{i}} \beta_{i k}^{\psi}\left(u^{\varepsilon}(t, x)\right)=\sqrt{\psi\left(u^{\varepsilon}(t, x)\right)} \sum_{i=1}^{d} \partial_{x_{i}} \beta_{i k}\left(u^{\varepsilon}(t, x)\right) \text { a.e. }
$$

Notice that, by a strong-weak limit, the right-hand side converges weakly in $L^{2}$ to

$$
\sqrt{\psi(u(t, x))} \sum_{i=1}^{d} \partial_{x_{i}} \beta_{i k}(u(t, x)) .
$$

Also, the left-hand side converges weakly in $L^{2}$ to

$$
\sum_{i=1}^{d} \partial_{x_{i}} \beta_{i k}^{\psi}(u(t, x)) .
$$

Therefore, we obtain property (ii) of Definition 2.1,

$$
\sqrt{\psi(u(t, x))} \sum_{i=1}^{d} \partial_{x_{i}} \beta_{i k}(u(t, x))=\sum_{i=1}^{d} \partial_{x_{i}} \beta_{i k}^{\psi}(u(t, x)) \text { a.e. }
$$

We may also pass to the limit in Eq. (2.1). Since, recalling the definition (2.6), $n(t, x, \xi) \leqslant w-\lim n_{\varepsilon}(t, x, \xi)$, we obtain that $u(t, x)$ satisfies Eq. (2.12) in $\mathcal{D}^{\prime}$ for some nonnegative measures $m(t, x, \xi)$ and $n(t, x, \xi)$ satisfying (2.19) and (2.20) as the argument in Section 2. Therefore, we have proved that $u \in C\left([0, \infty) ; L^{1}\left(\mathbb{R}^{d}\right)\right)$ is an entropy solution and Step 1 is completed.

Step 2. For the general case $u_{0} \in L^{1}$, we prove the existence of a kinetic solution directly. The same argument allows to build an entropy solution when $u_{0} \in L^{\infty}\left(\mathbb{R}^{d}\right)$.

Approximate $u_{0}(x)$ by $u_{0}^{\varepsilon} \in W^{2,1} \cap H^{1} \cap L^{\infty}\left(\mathbb{R}^{d}\right)$ such that

$$
u_{0}^{\varepsilon} \rightarrow u_{0}, \quad L^{1}\left(\mathbb{R}^{d}\right) .
$$

Then there exists a global entropy solution $u^{\varepsilon} \in C\left([0, \infty) ; L^{1}\left(\mathbb{R}^{d}\right)\right)$ of (1.1) and (1.2) with initial data $u_{0}^{\varepsilon}(x)$ for each $\varepsilon>0$. Using the contraction property of Theorem 1.1,

$$
\left\|u^{\varepsilon_{1}}-u^{\varepsilon_{2}}\right\|_{C\left([0, \infty) ; L^{1}\left(\mathbb{R}^{d}\right)\right)} \leqslant\left\|u_{0}^{\varepsilon_{1}}-u_{0}^{\varepsilon_{2}}\right\|_{L^{1}\left(\mathbb{R}^{d}\right)} \rightarrow 0, \quad \text { when } \varepsilon_{1}, \varepsilon_{2} \rightarrow 0 .
$$

Therefore, $\left\{u^{\varepsilon}\right\}$ is a Cauchy sequence and there exists $u \in C\left([0, \infty) ; L^{1}\left(\mathbb{R}^{d}\right)\right)$ such that

$$
u^{\varepsilon}(t, x) \rightarrow u(t, x), \quad \text { in } C\left([0, \infty) ; L^{1}\left(\mathbb{R}^{d}\right)\right) \text { when } \varepsilon \rightarrow 0,
$$

which implies the convergence of $u^{\varepsilon}(t, x)$ a.e. 
For any $\Psi$ as before, we have

$$
\sum_{k=1}^{K} \int_{0}^{\infty} \int_{\mathbb{R}^{d}} \psi\left(u^{\varepsilon}\right)\left(\sum_{i=1}^{d} \partial_{x} \beta_{i k}\left(u^{\varepsilon}\right)\right)^{2} \mathrm{~d} t \mathrm{~d} x \leqslant \int_{\mathbb{R}^{d}} \Psi\left(u_{0}^{\varepsilon}\right) \mathrm{d} x \leqslant C<\infty
$$

which implies

$$
\int_{0}^{\infty} \int_{\mathbb{R}^{d}}\left(\sum_{i=1}^{d} \partial_{x} \beta_{i k}^{\psi}\left(u^{\varepsilon}\right)\right)^{2} \mathrm{~d} t \mathrm{~d} x \leqslant C<\infty .
$$

On the other hand, for any two nonnegative functions $\psi_{1}, \psi_{2} \in \mathcal{D}(\mathbb{R})$,

$$
\sqrt{\psi_{1}\left(u^{\varepsilon}(t, x)\right)} \sum_{i=1}^{d} \partial_{x_{i}} \beta_{i k}^{\psi_{2}}\left(u^{\varepsilon}(t, x)\right)=\sum_{i=1}^{d} \partial_{x_{i}} \beta_{i k}^{\psi_{1} \psi_{2}}\left(u^{\varepsilon}(t, x)\right) \text { a.e. }
$$

These facts imply that the limit function $u(t, x)$ satisfies

$$
\sum_{i=1}^{d} \partial_{x_{i}} \beta_{i k}^{\psi}(u) \in L^{2}\left([0, \infty) \times \mathbb{R}^{d}\right)
$$

and

$$
\sqrt{\psi_{1}(u(t, x))} \sum_{i=1}^{d} \partial_{x_{i}} \beta_{i k}^{\psi_{2}}(u(t, x))=\sum_{i=1}^{d} \partial_{x_{i}} \beta_{i k}^{\psi_{1} \psi_{2}}(u(t, x)) \text { a.e. }
$$

The other properties in Definition 2.2 of kinetic solutions follow as in Step 1. Then the function $u(t, x)$ is a kinetic solution.

The contraction property and the argument in Section 5 for kinetic solutions at $t=0$ imply that the kinetic solution is stable in $L^{1}$, hence is unique.

This completes the proof of Theorem 1.2.

\section{Acknowledgements}

Gui-Qiang Chen's research was supported in part by the National Science Foundation under Grants DMS-9971793, INT-9987378, and INT-9726215.

\section{Appendix A}

In this appendix, we explain why a simplification occurs for diagonal diffusion matrices $a_{i j}(u)=0$ for $i \neq j$. Namely, we do not need to assume the identities (2.10) or (2.18) for diagonal diffusion matrices; they are always true with the only $L^{2}$ assumptions stated in (i) of Definition 2.1 or 2.2. This comes from some classical equalities in Sobolev spaces where very particular cancellations occur that cannot be true in the nondiagonal case. 
Proposition. - Let $\sigma \in L_{\text {loc }}^{\infty}(\mathbb{R}), \sigma(u) \geqslant 0$, and $\psi \in C_{0}(\mathbb{R})$. Set $\beta(u)=\int_{0}^{u} \sigma(\xi) \mathrm{d} \xi$ and $\beta^{\psi}(u)=\int_{0}^{u} \psi(\xi) \sigma(\xi) \mathrm{d} \xi$. Then, for any function $u \in L_{\mathrm{loc}}^{1}\left(\mathbb{R}^{d}\right)$ such that $\beta(u) \in$ $H_{\mathrm{loc}}^{1}\left(\mathbb{R}^{d}\right)$,

$$
\psi(u(x)) \partial_{x_{i}} \beta(u(x))=\partial_{x_{i}} \beta^{\psi}(u(x)), \quad \text { for } 1 \leqslant i \leqslant d .
$$

We do not give a detailed proof of this result that follows from classical analysis of Sobolev spaces. The reason why this proposition holds is that, setting $v=\beta(u)$, the identity reads

$$
\psi\left(\beta^{-1}(v)\right) \frac{\partial}{\partial x_{i}} v=\frac{\partial}{\partial x_{i}} \Psi_{\beta}(v),
$$

in $L_{\text {loc }}^{2}$, with $\Psi_{\beta}^{\prime}(\cdot)=\psi\left(\beta^{-1}(\cdot)\right)$ away from the countable points where $\beta^{-1}(\cdot)$ is discontinuous. However, at these points, $v$ is constant and thus $\frac{\partial}{\partial x_{i}} \Psi_{\beta}(v)=\frac{\partial}{\partial x_{i}} v=0$, see Gilbarg and Trudinger [15]. An argument based on regularizing $v$ and $\beta^{-1}(\cdot)$ can also prove the result.

Also see Bouchut [2] for a more difficult case for $u \in B V$; see also a similar statement, not necessarily stated as such, in Carrillo [6] and Eymard Gallouet and Herbin [13].

\section{REFERENCES}

[1] P. Bénilan, J. Carrillo, P. Wittbold, Renormalized entropy solutions of scalar conservation laws, Ann. Sc. Norm. Sup. Pisa 29 (2000) 313-327.

[2] F. Bouchut, Renormalized solutions to the Vlasov equation with coefficients of bounded variation, Arch. Ration. Mech. Anal. 157 (2001) 75-90.

[3] Y. Brenier, Résolution d'équations d'évolution quasilinéaires en dimensions $\mathrm{N}$ d'espace à l'aide d'équations linéaires en dimensions $N+1$, J. Differential Equations 50(3) (1982) 375-390.

[4] H. Brézis, M.G. Crandall, Uniqueness of solutions of the initial-value problem for $u_{t}-$ $\Delta \varphi(u)=0$, J. Math. Pure Appl. (9) 58 (2) (1979) 153-163.

[5] M.C. Bustos, F. Concha, R. Bürger, E.M. Tory, Sedimentation and Thickening: Phenomenological Foundation and Mathematical Theory, Kluwer Academic, Dordrecht, 1999.

[6] J. Carrillo, Entropy solutions for nonlinear degenerate problems, Arch. Rational Mech. Anal. 147 (1999) 269-361.

[7] G. Chavent, J. Jaffre, Mathematical Models and Finite Elements for Reservoir Simulation, North Holland, Amsterdam, 1986.

[8] G.-Q. Chen, E. DiBenedetto, Stability of entropy solutions to the Cauchy problem for a class of nonlinear hyperbolic-parabolic equations, SIAM J. Math. Anal. 33 (2001) 751-762.

[9] B. Cockburn, C. Dawson, Some extensions of the local discontinuous Galerkin method for convection-diffusion equations in multidimension, in: MAFELAP 1999 (Uxbridge), in: The Mathematics of Finite Elements and Applications, Vol. 10, Elsevier, Oxford, 1999, pp. 225-238.

[10] E. DiBenedetto, Continuity of weak solutions to certain singular parabolic equations, Ann. Mat. Pura Appl. 130 (1982) 131-176.

[11] J. Douglis, T. Dupont, R. Ewing, Incomplete iteration for time-stepping a Galerkin method for a quasilinear parabolic problem, SIAM J. Numer. Anal. 16 (1979) 503-522.

[12] M.S. Espedal, A. Fasano, A. Mikelić, Filtration in Porous Media and Industrial Applications, in: Lecture Notes in Math., Vol. 1734, Springer-Verlag, Berlin, 2000. 
[13] R. Eymard, T. Gallouët, R. Herbin, Existence and uniqueness of the entropy solution to a nonlinear hyperbolic equation, Chinese Ann. Math. Ser. B 16 (1995) 1-14.

[14] R. Eymard, T. Gallouët, R. Herbin, A. Michel, Convergence of a finite volume scheme for nonlinear degenerate parabolic equations, Preprint, 2001.

[15] D. Gilbarg, N. Trudinger, Elliptic Partial Differential Equations of Second Order, 2nd Edition, Springer-Verlag, Berlin, 1983.

[16] B.H. Gilding, Improved theory for a nonlinear degenerate parabolic equation, Ann. Scuola Norm. Sup. Pisa Cl. Sci. 16 (4) (1989) 165-224.

[17] K.H. Karlsen, N.H. Risebro, On convergence of finite difference schemes for viscous and inviscid conservation laws with rough coefficients, $M^{2}$ AN 35 (2) (2001) 239-270.

[18] S. Kruzhkov, First order quasilinear equations with several space variables, Mat. Sbornik 123 (1970) 228-255; Engl. Transl.: Math. USSR Sb. 10 (1970) 217-273.

[19] P.-L. Lions, B. Perthame, E. Tadmor, Formulation cinétique des lois de conservation scalaires multidimensionnelles, C. R. Acad. Sci. Paris, Série I Math. 312 (1991) 97-102.

[20] P.-L. Lions, B. Perthame, E. Tadmor, A kinetic formulation of multidimensional scalar conservation laws and related equations, J. Amer. Math. Soc. 7 (1994) 169-191.

[21] B. Perthame, Uniqueness and error estimates in first order quasilinear conservation laws via the kinetic entropy defect measure, J. Math. Pure Appl. 77 (1998) 1055-1064.

[22] B. Perthame, Kinetic Formulations of Conservation Laws, Oxford Univ. Press, Oxford (to appear).

[23] B. Perthame, F. Bouchut, Kruzhkov's estimates for scalar conservation laws revisited, Trans. Amer. Math. Soc. 350 (1998) 2847-2870.

[24] A.I. Volpert, S.I. Hudjaev, Cauchy's problem for degenerate second order quasilinear parabolic equations, Mat. Sbornik 78 (120) (1969) 374-396; Engl. Transl.: Math. USSR Sb. 7 (3) (1969) 365-387. 